\title{
ACCURACY AND REPRODUCIBILITY OF 3D PRINTED DIES VERSUS STONE DIES
}

\author{
Aliaa Mahrous* and Inass Aboulmagd ${ }^{* *}$
}

\begin{abstract}
Statement of the problem: researches studying the accuracy of the 3D printed dies were very rare.

Aim: The aim of the current study was to assess the difference in accuracy and reproducibility of the three dimensionally printed dies versus the stone dies.

Materials\& Methods: A maxillary typodont (D13PP-TR.1; Nissan, Kyoto, Japan) model with missing upper 1st molar was prepared as master model. Two types of dies were obtained from the master model ( $\mathrm{n}=6$ ). Group 1 was the conventional stone dies using type IV stone (GC FujiRock EP; GC) while group 2 representing the 3D printed dies (Dent2 Mogassam LLC Co. Egypt, Cairo) using resin (industrial blended resin, Funtodo Co. Netherlands). All working models were scanned (Open Technologies 3D scanner, Brescia, Italy) and STL file was superimposed on the STL file of the master model with the best-fit alignment method to analyze the volumetric changes and color maps were provided using 3Shape 3D software software. The mean average distance between the master dies and the working dies in all groups was recorded. Data were fed to the computer and analyzed using IBM SPSS software package version 20.0. Quantitative data were explored for normality using Kolmogorov-Smirnov and Shapiro-Wilk tests.
\end{abstract}

Results: There were no statistically significant differences in volumetric discrepancies between stone model $(0.186 \pm 0.231) \mathrm{mm}^{3}$ as a $($ mean $\pm \mathrm{SD})$ and $3 \mathrm{D}$ printed model $(0.176 \pm 0.211) \mathrm{mm}^{3}$ as a mean \pm SD.

Conclusion: Digital work flow can eventually replace the conventional techniques with minimal volumetric discrepancies in 3D printed dies.

KEYWORDS: dimensional accuracy, stone dies, rapid prototyping, 3D printed dies, digital dentistry, digital light processing, best-fit alignment, color map

\footnotetext{
* Lecturer Fixed Prosthodontics Department, Faculty of Dentistry, Fayoum University, Egypt.

** Lecturer oral Radiology Department, faculty of Dentistry, Fayoum University, Egypt.
} 


\section{INTRODUCTION}

Computer-based engineering is being incorporated in restorative dentistry using digital processes from intraoral scanning (i.e., image capture), through fabrication of the definitive restoration. These processes have been termed the "digital workflow" Using developed algorithms. The digital workflow can be entirely virtual or may include traditional technologies. In a completely digital workflow, the abutment tooth is recorded with a laser-based scanning device, a virtual cast developed, the restoration designed virtually and finally the digital image saved in an STL file format. A multi-axis milling machine or a rapid prototyping (RP) device as 3D printer can be used to produce the restoration from the STL file ${ }^{(1 \& 2)}$. The virtual casts also have the efficiency, convenience and durability with elimination of the need for physical storage area associated with traditional cast ${ }^{(3-6)}$.

However, in a second algorithm, when the need for this physical model is a must for designing, STL file can be converted in a resin model using 3D printer technology as the most appreciate method for cast production from digital image with the high precision and quality ${ }^{(7 \& 8)}$.

The 3D printing technology can be considered relatively new as it was developed in late 1980s, and has captured the public imagination with many modalities in use today. The 3D (dimensionally) printing is a term describing an additive manufacturing (AM) approach of building an object by layers, one layer per time which is repeated multiple to form the final object. It can be also referred to as rapid prototyping ${ }^{(9)}$.

There are different technologies at the market with many advantages and limitations. $1^{\text {st }}$ Steriolithography (SLA, SL) which is astereolithography apparatus uses a scanning laser to build parts one layer at a time, in a vat of lightcured photopolymer resin that finally UV cured in oven. Photojet Light sensitive polymer is another technology based on jetting onto a build platform from an inkjet type print-head, and cured layer by layer on an incrementaly descending platform. DLP (digital light processing) is using liquid resin which is cured layer by layer by a projector light source. The object is built upside down on an incrementally elevating platform ${ }^{(9-12)}$.

Type IV dental stone have been used as the material of choice in model fabrication even with its drawbacks as low wear resistance and setting expansion $^{(13)}$. It's still more popular than epoxy and polyurethane resin. The accuracy of impressions and casts are a key success of the accuracy of fixed restoration $^{(14 \& 15)}$.

The aim of the present study was to assess the difference in accuracy and reproducibility of the three dimensionally printed dies versus the stone dies using superimposition of the master cast with the stone cast and 3D printed cast. The null hypothesis was that there were no difference in accuracy and reproducibility between the stone cast and $3 \mathrm{D}$ printed cast.

\section{MATERIAL \& METHODS}

\section{Dies preparation of typodont model}

A maxillary typodont (D13PP-TR.1; Nissan, Kyoto, Japan) model with missing upper $1^{\text {st }}$ molar, prepared upper $2^{\text {nd }}$ premolar and $2^{\text {nd }}$ molar was selected as a master cast for the current study. The abutment teeth were prepared with the following features; deep chamfer finish line with average 1.5 $\mathrm{mm}$ axial reduction, $2 \mathrm{~mm}$ occlusal reduction and total convergence angle of $12^{\circ}$.

Two test groups of the working model were obtained from the master model with six samples of each tested group $(n=6)$. The tested groups were; group I representing the conventional stone cast while group II representing the $3 \mathrm{D}$ printed cast.

\section{Stone model fabrication}

The typodont model was duplicated and customized acrylic trays (Kilgore Int. Inc., Cold water, mish, USA) were fabricated on the duplicated 
master model to ensure even impression material thickness of 2 to $4 \mathrm{~mm}$.

The impression of the master model was taken using addition silicon polyvinyl siloxane (Aquasil LV, Putty/Light Body, Dentsply, Germany) one step technique and repeated for six times. All impressions were allowed to stay for three times longer than the recommended time by the manufacturer to ensure the polymerization at room temperature.

After impression removal, it was inspected under magnifying lens for any defects or tearing before it was rinsed with running water and dried. Impression was sprayed with approximately 0.5 $\mathrm{ml}$ surfactant (Debubblizer Surfactant; Almore International Inc) which decrease the surface tension to improve the final cast quality. Dental stone type IV (GC FujiRock EP; GC) was mixed under vacuum (Twister Evolution; Renfert) following the manufacturer's instruction and poured with vibration. The working cast was left for complete setting for 45 minutes before its removal from the impression. The procedure was repeated for six times to produce six working models.

\section{D printed model fabrication}

Regarding the 3Dprinted models; virtual impressions were made from master model by a single experienced operator using light 3D scanners with Blue LED technology (Open Technologies 3D scanner, Brescia, Italy). The scanned files were converted to stereolithography (STL files) which is the format needed to produce physical 3D printed models using DLP (digital light processing) technique. 3D printer (Dent2 Mogassam LLC Co. Egypt, Cairo) with XY resolution of $30-50 \mu \mathrm{m}$, layer thickness $25 \mu \mathrm{m}$ and speed $3 \mathrm{~cm}$ hhour was used to produce six 3D working models from resin (industrial blended resin, Funtodo Co. Netherlands).

\section{Best-fit alignment}

All working models (stone models \& 3D printed models) were scanned using structured light 3D scanner (Open Technologies 3D scanner, Brescia, Italy) with the same operator. STL file of the typodont (master model) (Figure 1) was superimposed on the resulted STL file of each working model using special software (3Shape3D) utilizing the best-fit alignment method to analyze the volumetric changes (Figure: $2 \mathrm{a} \& \mathrm{~b}$ ).

Best-fit alignment ${ }^{(16)}$ is a special method in software that computing all possible orientations and selecting the one with the best object to object penetration. It can be used to compare the minimum distance variation from every measured point in master model with the points of the examined model with interactive least square fitting algorithm.

Color maps of the best-fit method were provided using 3Shape 3D software, which automatically measured the mean average distance between the master model and the working models in all groups. The mean average distance is the absolute value of the outline discrepancy between master model and working models.

\section{Statistical analysis of the data}

Data were fed to the computer and analyzed using IBM SPSS software package version 20.0. Quantitative data were explored for normality using Kolmogorov-Smirnov and Shapiro-Wilk tests. All data showed parametric distribution. Data were presented as mean and standard deviation (SD) values. Student's t-test was used to compare between the two groups. Significance of the obtained results was judged at the $5 \%$ level.

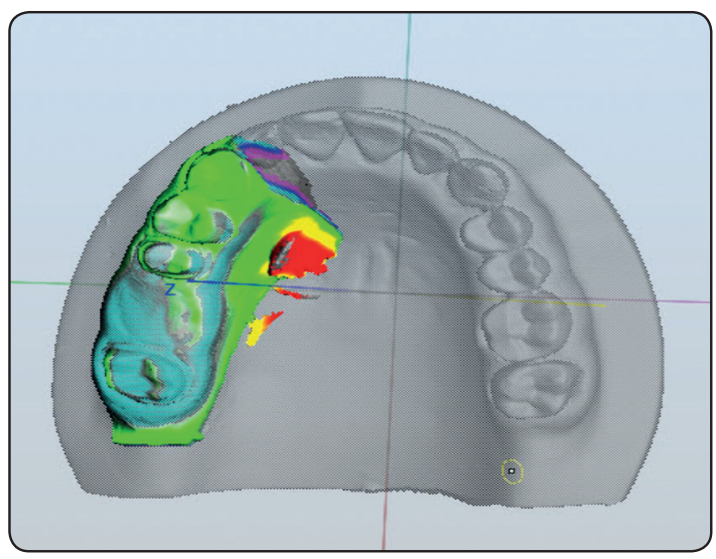

Fig. (1) Registration of the master model. 


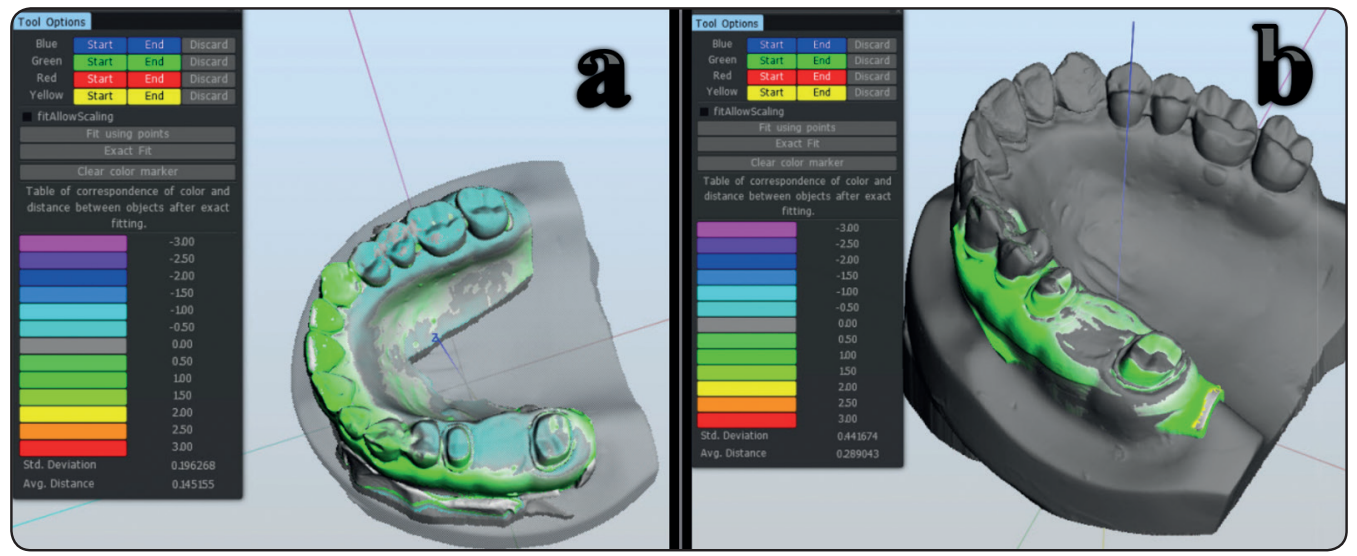

Fig. (2) STL files superimposition of stone model (a) \& 3D printed (b) models.

\section{RESULTS}

The superimposition of the tested groups showed all negative and positive with total volumetric discrepancies between the master dies "registration" (Figure1) with stone dies and 3D printed dies (Figure $2 \mathrm{a} \& \mathrm{~b}$ ). Discrepancies between the master model and the tested groups were analyzed with the color map where positive discrepancies (larger than master model) ranged from the grey color to orange color and negative discrepancies (smaller than master model) ranging from grey to pink color (Figure $3 \mathrm{a} \& \mathrm{~b}$ ).
The average in color maps of the stone casts showed gray (0.00 discrepancy) except for buccal axial walls and the buccal finish line with light green color (0.5) indicating expansion of these areas. While regarding the $3 \mathrm{D}$ printed models, the grey color (0.0 discrepancy) was covering the majority of the map except for the occlusal surface showed light blue (-0.5) indicating contraction.

The statistical analysis of the data retrieved from both groups for volumetric discrepancies showed that $0.186 \pm 0.231 \mathrm{~mm}^{3}$ as a mean $\pm \mathrm{SD}$ of the stone group while in 3D printed group was $0.176 \pm 0.211$ $\mathrm{mm}^{3}$ as a mean $\pm \mathrm{SD}$ with no statistically significant differences (Table 1, Figure 4).

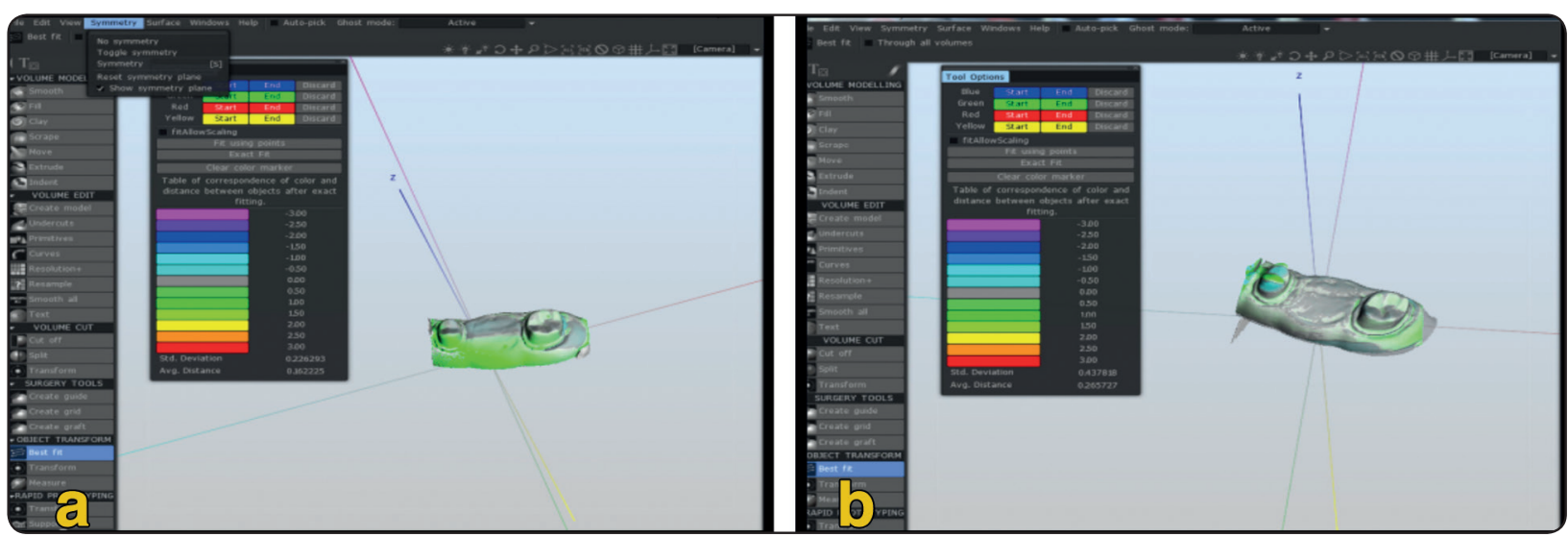

Fig. (3) Color coded map made from best-fit alignment of the stone dies(a) and 3D printed dies (b). 
TABLE (1) Volumetric differences made on stone dies and 3D printed dies

\begin{tabular}{|c|c|c|c|}
\hline & $\begin{array}{c}\text { Group I Stone die } \\
(\mathrm{n}=6)\end{array}$ & $\begin{array}{c}\text { Group II 3D printed die } \\
(\mathrm{n}=6)\end{array}$ & p-Value $^{*}$ \\
\hline Volume $\left(\mathrm{mm}^{3}\right)$ & $0.186 \pm 0.231$ & $0.176 \pm 0.211$ & 0.76 \\
\hline
\end{tabular}

Qualitative data were described using number and percent, while normally quantitative data was expressed in mean $\pm S D$, abnormally distributed data was expressed in median (Min. - Max.) $\quad$ *: Statistically significant at $p \leq 0.05$

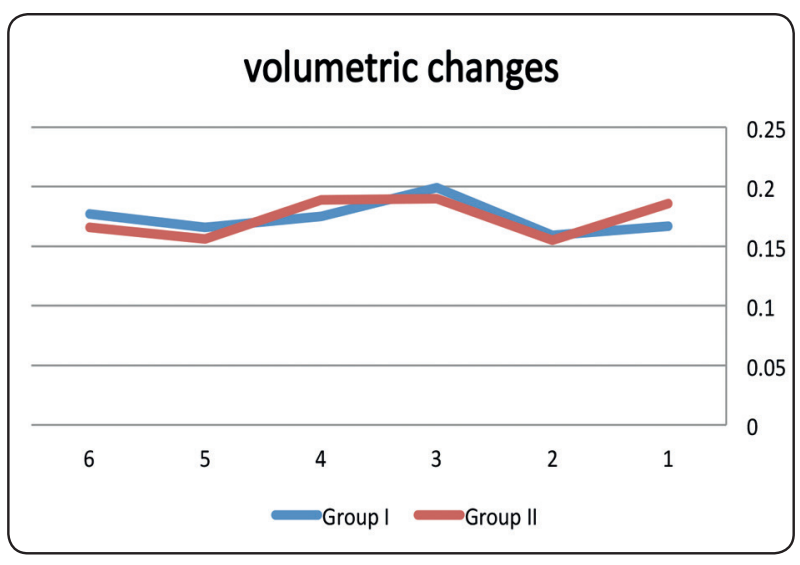

Fig. (4) Line chart showing volumetric changes of master dies $\&$ working dies.printed (b) models.

\section{DISCUSSION}

Construction of prosthetic restorations with accurate internal fit and good marginal adaptation were mainly defendant on producing dental models with high accurate dimensions simulating original teeth to decrease the discrepancy ${ }^{(14)}$.

Discrepancy is a term defined the difference in dimensions between both casts (master model \& working model) measured by superimposition. Accuracy was a term describing how close the data were to the master model and reproducibility examined the consistency of the casts to each other ${ }^{(17)}$.

The results of the present study showed that there were no statistically significant differences of the volumetric changes between both tested groups (stone cast \& 3D printed cast) (Table 1, Figure 4). The null hypothesis of our study was accepted and the results of discrepancies were within the clinically accepted range $\mathrm{e}^{(1 \& 18)}$.
These were in accordance with Cho et al ${ }^{(17)}$ who mentioned that there were no statistically significant differences between stone model and digital models. However, the working dies of traditional stone were more accurate than the dies from digital approach. In addition to Ender et al ${ }^{(19)}$ who mentioned that there the accuracy of conventional and digital impression were not differ. While on the other side, Park et $\mathrm{al}^{(16)}$ reported that die fabrication by $3 \mathrm{D}$ printer is not as reliable as the stone die as the conventional stone dies have less volumetric changes than 3D printed dies.

It is well known that vinyl siloxanether impression material have better dimensional accuracy than either polyether or polyvinyl siloxane $^{(20,21)}$. Moreover, the setting expansion of Type IV dental stones is intended to match the polymerization shrinkage of vinyl siloxanether materials $^{(22)}$ but the dimensions of the resulted working cast still have oversize than the original tooth $^{(21)}$. These were in accordance with our results as the stone dies showed positive discrepancy (0.5) at the buccal wall and buccal finish line indicating expansion of these areas (Figure 3a).

As the continuous progress in digital technology and $\mathrm{CAD} / \mathrm{CAM}$, the need for conventional impression and models decreased and digital impression using digital scanners to generate a digital dataset increased. These digital dataset (STL) files sometimes not enough to substitute the physical model. Model fabrication using 3D printing is the commonly suitable technique here ${ }^{(17)}$. 
The model fabrication using the additive approach of 3D printing will be influenced by the applied technology of the machine. These technologies can cause dimensional differences with subsequent effect on accuracy of the produced models as a result of many factors such as; the deposited minimal layer thickness and shrinkage of the selected material during building or postcuring ${ }^{(11 \& 23)}$.

There were no study has specified the clinically acceptable differences between the die and original model. However, several studies ${ }^{(11,16 \& 24)}$ comparing conventional plaster models with dental replica models reported clinically acceptable differences of less than $0.25 \mathrm{~mm}$ for being similar to the tolerances for manual measurements ${ }^{(17)}$.

A study by Lee et al ${ }^{(25)}$ confirmed that 3Dprinted approach could be used in treatment requiring high accuracy as orthodontic or fixed prosthodontics especially with FDM $(0.047 \mathrm{~mm})$ \& polyjet $(0.038 \mathrm{~mm})$ technology with total dimensional difference within the clinically accepted range. Although, the dies fabricated using PolyJet technology had statistically significant higher accuracy but both technologies have a difference within the clinically accepted range ${ }^{(18)}$. The difference in dimensions between both technologies may be explained by difference in layer thickness (FDM: $0.330 \mathrm{~mm}$, PolyJet: $0.016 \mathrm{~mm})^{(26)}$.

The same study of Lee et al ${ }^{(25)}$ continued the analysis using color map of the best-fit alignment found that discrepancies were seen at bifurcation area and occlusal surface. Due to difficulty of scanning curved and complicated parts accurately. Also, STL file formation may be distorted during conversion and manipulation ${ }^{(27)}$. These were greatly in accordance with our results as the discrepancies were mostly located at curved occlusal surface and finish line areas (Figure $3 \mathrm{~b}$ ). Bibb et $\mathrm{al}^{(28)}$ mentioned that the accuracy of any light scanner will be greater in straight surfaces rather than curved surface as light travels in straight lines.
Finally, the reasonable cost of 3D printers and the continuous progress in this technology with the ability to incorporate by many dentists into clinical work daily, the need for conventional approach decreased. 3D printed models can be used for treatment planning or in surgical procedures ${ }^{(16)}$.

Several limitations were considered in the present study as using single type of conventional impression, one type of 3D printed machine and printed material. Also, the use of typodont model with elimination of the actual similarity to oral cavity as no saliva contamination and taking impression at room temperature were limitations of the study.

Further researches were required evaluating different span dimensions with other preparation dimensions with different combinations of 3D printer and different printing materials.

\section{CONCLUSION:}

Within the limitations of the present study,

1- Digital work flow can eventually replace the conventional techniques.

2- 3D printed models with volumetric discrepancies within the clinically accepted range.

Clinical implication: 3D printed models with digital impression can be used with adequate accuracy and reproducibility as conventional methods

\section{REFRANCES}

1. Mahmoud Serag,Talal al Nassar, Dane Avondoglio\& Saul Weiner, A comparative study of the accuracy of dies made from digital intraoral scanning vs. elastic impressions: an in vitro study. J Prosthodont 2018: 27:88-93

2. Christensen GJ: Impressions are changing: deciding on conventional, digital or digital plus in-office milling. J Am Dent Assoc 2009;140:1301-1304.

3. McGuinness NJ, Stephens CD. Storage of orthodontic study models in hospital units in the U.K. Br J Orthod 1992;19:227-32. 
4. Ayoub AF, Rehab M, O’Neil M, Khambay B, Ju X, Barbenel J, et al. A novel approach for planning orthognathic surgery: the integration of dental casts into three-dimensional printed mandibular models. Int J Oral Maxillofac Surg 2014;43:454-9.

5. Soares PV, de Almeida Milito G, Pereira FA, Reis BR, Soares CJ, de Sousa Menezes M. Rapid prototyping and 3D-virtual models for operative dentistry education in Brazil. J Dent Educ 2013;77:358-63.

6. Scherer MD. A contemporary approach to intraoral optical scanning and inoffice 3-D printing. Dent Today 2015;34:46-7.

7. Sun J, Zhang FQ. The application of rapid prototyping in prosthodontics. J Prosthodont 2012;21:641-4.

8. Sarah Bukhari, Brian J. Goodacre, Abdulaziz AlHelal, Mathew T. Kattadiyil, Paul M. Richardson, Three-dimensional printing in contemporary fixed prosthodontics: A technique article. J Prosthet Dent 2018;119:530-4.

9. Hull CW. Apparatus for production of three-dimensional objects by stereolithography.1986:US Patent 4575330.

10. Dawood, Marti Marti, Sauret-Jackson, Darwood. 3D printing in dentistry. British Dent J 2015; 219 (11):521-530.

11. Murugesan K, Anandapandian PA, Sharma SK, Kumar MV. Comparative evaluation of dimensional and surface detail accuracy of models produce by three different rapid prototype techniques. J Indian Prosthodont Soc 2012;12:16-20.

12. Xie R, Li D, Chao S. An inexpensive sterolithography technology with high power UV-LED light. Rapid Prototyping J 2011;17:441-50.

13. Kenyon BJ, Hagge MS, Leknius C, Daniels WC, Weed ST. Dimensional accuracy of 7 die materials. J Prosthodont 2005;14:25-31

14. Persson ASK, Andersson M, Oden A, Sandborgh-Englund G. Computer aided analysis of digitized dental stone replicas by dental CAD/CAM technology. Dent Mater 2008;24:1123-30.

15. Prisco R, Cozzolino G, Vigolo P. Dimensional accuracy of an epoxy die material using different polymerization methods. J Prosthodont 2009;18:156-61.

16. Mid-Eum Park, Soo-Yeon Shin.Three-dimensional comparative study on the accuracy and reproducibility of dental casts fabricated by 3D printers. J Prosthet Dent 2018.
17. Seok-Hwan Cho, Oliver Schaefer, Geoffrey A. Thompson, Arndt Guentsch. Comparison of accuracy and reproducibility of casts made by digital and conventional methods. J Prosthet Dent 2015;113(4):310-315.

18. Hazeveld A, Huddleston Slater JJ, Ren Y. Accuracy and reproducibility of dental replica models reconstructed by different rapid prototyping techniques. Am J Orthod Dentofacial Orthop 2014;145:108-15.

19. Ender A, Mehl A. Full arch scans: conventional versus digital impressions: An in-vitro study. Int J Comput Dent 2011;14:11-21.

20. Thongthammachat S, Moore BK, Barco MT, Hovijitra S, Brown DT, Andre CJ. Dimensional accuracy of dental casts: Influence of tray material, impression material, and time. J Prosthodont 2002:98-108.

21. Stober T, Johnson GH, Schmitter M. Accuracy of the newly formulated vinyl siloxanether elastomeric impression material. J Prosthet Dent 2010;103:228-39.

22. Schaefer O, Schmidt M, Goebel R, Kuepper H. Qualitative and quantitative three-dimensional accuracy of a single tooth captured by elastomeric impression materials: an in vitro study. J Prosthet Dent. 2012;108:165-72.

23. Barker TM, Earwaker WJ, Lisle DA. Accuracy of stereolithographic models of human anatomy. Australas Radiol 1994;38:106-11.

24. Hiroqaki Y, Sohmura T, Satoh H, Takahashi J, Takada K. Complete 3-D reconstruction of dental cast shape using perceptual group. IEEE Trans Med Imaging 2001;20:1093101.

25. Lee KY, Cho JW, Chang NY, Chae JM, Kang KH, Kim SC, Cho JH. Accuracy of three-dimensional printing for manufacturing replica teeth. Korean J Orthod 2015;45(5):217225.

26. Keating AP, Knox J, Bibb R, Zhurov AI. A comparison of plaster, digital and reconstructed study model accuracy. J Orthod 2008;35:191-201.

27. Cheah CM, Chua CK, Tan KH, Teo CK. Integration of laser surface digitizing with CAD/CAM techniques for developing facial prostheses. Part 1: Design and fabrication of prosthesis replicas. Int J Prosthodont 2003;16:435-41. rthod. 2015 Sep;45(5):217-25.

28. Bibb R, Freeman P, Brown R, Sugar A, Evans P, Bocca A. An investigation of three-dimensional scanning of human body surfaces and its use in the design and manufacture of prostheses. Proc Inst Mech Eng H 2000; 214:589-94. 